\title{
Cognitive and emotional regulation in adolescents and young women with eating disorders
}

Chiara Malagoli1, Pier Fabrizio Cerro2, CaterinaVecchiato2 and Maria Carmen Usai1

1Department of Education, University of Genoa, C.so Andrea Podestà 2, 16128 Genova, Italy; 2 Regional Centre for Eating Disorders and Adolescence; Centro Regionale per i disturbi del comportamento alimentare e dell'adolescenza Cdaa Asl 2, Via XXV Aprile 128 - Ospedale S. Corona (Pad. Racamier)Pietra Ligure Savona, Italy ;

E-mail: chiara.malagoli@edu.unige.it; cerro.p@alice.it; catevede@libero.it; maria.carmen.usai@unige.it

Corresponding author: Chiara Malagoli, Department of Education, University of Genoa, C.so Andrea Podestà 2, 16128 Genova, Italy.

Orcid: 0000-0001-6872-2315

E-mail: chiara.malagoli@edu.unige.it

Malagoli C., Cerro P.F, Vecchiato C., Usai M. C. (2020). Cognitive and emotional regulation in adolescents and young women with eating disorders. Eating and Weight Disorders - Studies on Anorexia, Bulimia and Obesity. doi: 10.1007/s40519-020-00859-x 


\title{
Cognitive and emotional regulation in adolescents and young women with eating disorders
}

\begin{abstract}
Background: Eating disorders (EDs) are associated with a reduced ability to regulate emotion and impulses during the lifespan. Working memory (WM) and executive functions (EF) are cognitive regulatory systems supported by networks involving the pre-frontal cortex. Studies in EDs found impaired functioning in these domains, showing an association between EDs and the reduced ability to control emotions and impulses.
\end{abstract}

Objective: Investigate EF in adolescents and young women with eating disorders (ED) using a quasi-experimental design, focusing on cognitive efficiency, emotional regulation (ER) and behavioural outcomes also taking into account pharmacological treatment and duration of illness.

Methods: A sample of 151 females belonging to two groups took part in this study. Twentysix girls and young women (Mage 22;8 years) with ED and 125 typically developed girls and young women (Mage 17;4 years) completed a battery of cognitive tasks (Go-No-Go, StopSignal task, Symmetry span, Reading Span) and the Youth Self-Report and the Difficulties in Emotional Regulation Scale Performance. A series of ANOVA with the Brown-Forsythe test was used to compare the groups

Results: Participants with ED and controls did not show significant differences in EF tasks, whereas differences between younger and older participants with ED emerged. Moreover, ER difficulties seem to be associated with mainly internalizing problems in EDs. Further analysis on the full ED sample did not reveal any significant differences associated with the disorder 
persistence. Considering pharmacological treatment effects over cognitive, emotional and behavioural measures emerged.

Conclusions: The present study documented no specific differences in EF between control and participants with EDs. Whereas important differences emerged in ER and behavioural outcomes perception in the clinical sample, together with a partial influence of pharmacological treatment.

Keywords: Eating disorders, cognitive processes, emotional regulation, behavioural outcomes, adolescence

Level of evidence: No level of evidence 


\section{Cognitive and emotional regulation in adolescents and young women with eating disorders}

Eating disorders (EDs) are associated with a reduced ability to regulate emotion and impulses during the lifespan [1]. Food assumption and appetite, defined as the motivational drive to obtain food, are subordinated by the basal regulation of hunger and fullness. It has been demonstrated how the interaction between the regulatory functions operated by the pre-frontal cortex together with sub-cortical activity in the brain has an important role in the aetiology and persistence of EDs, by analogy with what is documented in addictive behaviors.

Literature, in fact, documents how in patients diagnosed with EDs it was possible to find specific difficulties connected to cognitive processes supported by pre-frontal cortex, e.g. [2;3].

Working memory (WM) and executive functions (EF) are cognitive regulatory systems supported by networks involving the pre-frontal cortex. Most studies on adults with EDs found impaired functioning in these cognitive domains, showing an association between EDs and the reduced ability to control emotions and impulses $[4 ; 5]$. Despite these documented results in the existing literature, there is still a lack of a broader investigation concerning these possible fragility factors in adolescents and young adults. Due to the high sensitivity of these processes to both individual differences and environmental stimulation, a deeper knowledge of these topics would allow a better understanding of ED aetiology and contribute to clinical advancement, opening up a concrete possibility for individualized early treatment.

During adolescence, EF continues to develop in parallel with changes in synaptic density in the prefrontal brain regions [6]. In particular, inhibition and updating of WM, as core abilities of EF, e.g., [7], are shown to evolve throughout middle and late adolescence [8] and follow a protracted development into young-adulthood [9]. These processes significantly influence individual abilities to adapt to the context and manage environmental complexity. Individual differences in WM capacity are associated with a variety of cognitive and social outcomes, e.g., [10]. Dysfunctions in 


\section{Running Head: COGNITIVE AND EMOTIONAL REGULATON IN EATING DISORDERS}

EF correlate with risky behaviours and maladaptive outcomes already in pre-adolescence [11]. In particular, impulsiveness has been associated with non-adaptive behaviours: e.g. increased substance abuse, gambling [12], and self-injuries [13]. With regard to literature concerning individuals diagnosed with EDs, recent research has documented EF impairments [2] and particularly poor inhibitory control in binge eating, e.g. [14]. Adolescents who have a shorter experience of EDs also show deficits in EF [15] that persist after symptom remission [16, 17], suggesting that EF impairments are causal rather than consequential to EDs [18]. EDs are also characterized by difficulties in emotion regulation (ER), suggesting that emotion dysregulation may be a factor underlying their co-occurrence, e.g. [19]. EF and ER are complex cognitive features that interact to support one's ability to self-regulate in many aspects of every-day life [20]. Literature documents how difficulties in ER occur in a variety of conditions, as a consequence of trauma [21] or in association with severe clinical disorders [22]. Recent research has documented abnormalities in emotion generation/regulation in individuals with EDs [23; 24; 26; 27$]$.

The affect regulation model of EDs proposes that disordered eating behaviours are the result of the attempt to decrease negative emotions [28]. Emotion dysregulation seems to be an important predictor of binge eating and a core factor that may predict the onset of EDs (e.g. dietary restriction [29]). Individuals with EDs tend to rely on maladaptive strategies (e.g. emotion suppression/ avoidance [30]) and these strategies often translate into maladaptive behaviours (e.g., loss of control over eating [31]). Externalizing tendencies are also documented in EDs [32].

WM was recently found negatively associated with difficulties in two separate ER dimensions labeled ER knowledge and ER responses, referring to cognitive reappraisal and emotion-generative process respectively, but no significant and direct relationship with internalizing and externalizing self-reported difficulties emerged [33]. One explanation may be the absence of a clinical sample in which maladaptive outcomes, such as extreme externalizing and internalizing tendencies, are more strongly related to the aforementioned cognitive features. Even though some important research in 
the field has been conducted, an investigation into inhibition and WM in adolescence, which also considers difficulties in ER and behavioural tendencies in EDs, particularly investigating specific aspects of inhibition and WM and taking also into account the variable time, in terms of duration of illness and pharmacological treatment when present, is still lacking, as a great part of the literature is more focused on profiling EF in adulthood.

\section{The present study}

This study focuses on inhibition, WM, and emotional difficulties in adolescents and young women with EDs compared to a typically developing (TD) control group, using a quasiexperimental design. In the literature, whether these processes may be characterized by a different pattern of functioning compared to TD is still an open question. We expect the ED group to function differently in terms of inhibitory and WM efficiency in comparison to the TD group, and in terms of ER and behavioural outcomes as well. Also, we hypothesize that age influences performance in EF tasks, as a time effect in the development of these abilities is documented. The effects due to the duration of illness and effects of the eventual pharmacological treatment were also taken into account investigating inhibition, WM, ER and behavioural outcomes in the ED group.

\section{Method}

\section{Participants}

A final sample of 151 females belonging to two groups took part in this study. Twenty-six girls and young women (Mage 22;8 years, S.D. 7;3, range 15;0 - 44;5) with ED were included in two groups formed on the basis of the age range: a younger group (Adolescents; ED1) of 13 girls (Mage 17;9 years, S.D. 0;1, range 15;0 - 20;0) and an older group (Young Adults; ED2) of 13 women (Mage 28;5 years, S.D. 6;9, range 21;8 - 44;5). One-hundred-twenty-five TD girls and young women (Mage 
$17 ; 4$ years, S.D. 1;2, range 15;3 - 19;9) were included in the control group. Individuals in the clinical sample received a diagnosis of EDs (anorexia, bulimic syndromes, Other Specified Feeding or Eating Disorder - OSFED) according to DSM 5 and were recruited from two treatment centers in the north of Italy. All participants included in the clinical sample (for a complete description see Figure 1) received diagnosis throughout the diagnostic assessment and the administration of clinical questionnaires. TD participants were recruited from different high schools in the same area. None of them had a history of neurological impairment/developmental disabilities. Besides psychotherapeutic and medical support, participants with EDs attended a dedicated "module" of mindfulness practice in a weekly meeting of two hours during two months of intensive care hospitalization.

\section{Materials and Procedure}

We administered two 45-minute sessions in a quiet room provided by the school and the centers. The self-reports were filled autonomously by the participants.

Cognitive tasks (for an extensive description see [8, 33]).

Stop Signal task [34]. This task measures response inhibition and consists of two phases: a practice phase of 32 trials and an experimental phase of three blocks of 64 trials. In both phases, each trial starts with the presentation of the fixation sign, which is replaced by the primary-task stimulus after $250 \mathrm{msec}$. The stimulus remains on the screen until subjects respond or $1250 \mathrm{~ms}$ have elapsed. The interval between stimuli is $2000 \mathrm{~ms}$ and is independent of RT. During "stop" trials, a signal is presented after a variable Stop Signal Delay that is initially set at $250 \mathrm{~ms}$ and adjusted continuously with the staircase procedure. The dependent measure was the stop-signal reaction time SSRT index [35]. A split-half reliability with the Spearman-Brown formula was calculated (.84).

Go-No-Go task [36]. The Go-No-Go assesses the ability to stop an automatic response and required participants to press the spacebar when a given figure target was displayed and not to press 


\section{Running Head: COGNITIVE AND EMOTIONAL REGULATON IN EATING DISORDERS}

if any other figure was displayed (20\% of the stimuli). The task consisted of a practice phase of 20 trials and a test phase of 100 trials. The stimulus duration time was $1500 \mathrm{~ms}$. The dependent variable was accuracy at the No-Go trials. The Cronbach's alpha calculated on the TD group was .70.

Emotional Go-No-Go task [4; 37]. This type of Go-No Go measures the inhibition of an automatic response in a more emotional condition, using faces that express a particular emotion. The set of stimuli consisted of grey scale images of 10 adults (five males) posing three different expressions (happy, fearful and neutral). Visual angle was approximately $12^{\circ}$. Face stimuli were presented singly in the center of the screen. Subjects were instructed to press the space bar as fast as they could when a named expression was presented and to withhold pressing the space bar in case of a "no-go" condition (30\% of the trials). In each block, an emotional expression was always paired with a neutral expression. The test was composed of 6 randomized blocks with 50 randomized trials for each condition. Stimulus duration was $500 \mathrm{~ms}$ with 1000 . The dependent variable was accuracy at the No-Go trials. The Cronbach's alpha calculated on the TD group was .65 .

Symmetry Span task (SymmSpan [38]). This is a complex measure of WM capacity composed of two different tasks performed at the same time. The first one consists of recalling a sequence of squares that appear on the screen while the second one consists of judging if some figures are symmetrical or not. Participants are then instructed to recall the whole sequence of squares in the correct order and to maintain at least $85 \%$ accuracy in the symmetry task. The dependent variable was the absolute span score (SSPAN score): the sum of all perfectly recalled sets. A split-half reliability procedure was performed for this task. The Spearman-Brown coefficient for the TD sample was .91.

Reading Span task (RSPAN [39]). This span is structurally identical to the previous one but presents different stimuli. In fact, the tasks consist of recalling a sequence of letters that appear on 


\section{Running Head: COGNITIVE AND EMOTIONAL REGULATON IN EATING DISORDERS}

the screen while judging if some phrases make logical sense or not. The two tasks are clustered in sets (range two to seven). As mentioned in the previous task we used the sum of all perfectly recalled sets (RSPAN score). Participants were instructed to maintain at least $85 \%$ accuracy at the phrase problem task. A split-half reliability procedure was performed for this task. The SpearmanBrown coefficient was .91.

\section{Self-report measures}

Difficulties in Emotion Regulation Scale (DERS Italian version [40]). The DERS is a 36-item self-report measure developed to assess clinically relevant difficulties in ER. Items provide scores on six scales: Non-acceptance of Emotional Responses, Difficulties Engaging in Goal-Directed Behaviour, Impulse Control Difficulties, Lack of Emotional Awareness, Limited Access to Emotion Regulation Strategies, and Lack of Emotional Clarity. Participants have to indicate how often each item applies to them on a 5-point Likert-type frequency scale. Cronbach's alphas for each subscale range from .76 to .89 .

Youth Self-Report (YSR, 11-18 years [41]). It provides an assessment of the respondent's social/emotional functioning. The YSR comprises 112 items scored using a three-point frequency scale and provide scores on eight subscales clustered to identify the individual's externalizing (Rule-Breaking Behaviour and Aggressive Behaviour) or internalizing (Anxious/ Depressed, Withdrawn/Depressed, and Somatic Complaints) tendencies. Cronbach's alphas ranged from .70 to .78. The YSR was not administered to the ED2 group because these women were over the age indicated in the questionnaire regulations.

\section{Statistical Analyses}

Descriptive statistics and ANOVA with the Brown-Forsythe test was used to compare the groups, and the Tamhane test was performed as a post-hoc test. The Brown-Forsythe is a robust test based on the absolute differences within each group from the group median. A larger control group was enrolled to control individual variability factors using robust statistics [42]. Cohen's d for 


\section{Running Head: COGNITIVE AND EMOTIONAL REGULATON IN EATING DISORDERS}

groups with different sample sizes was used to compute the effect size of significant pairwise comparisons between groups. To summarize the information about ER obtained with the DERS scales, an exploratory factor analysis (EFA) using principal axis factoring with varimax rotation of the factor structure was executed.

\section{Results}

The ANOVA revealed a main effect of age, $\mathrm{F}(2,150)=30.981 \mathrm{p}<.001 \eta^{2}=.648$. The posthoc analysis showed that the ED2 were significantly older than both the control $(p<.001, d=4.704$ $[95 \% \mathrm{CI}=3.907-5.5])$ and the ED1 $(\mathrm{p}<.001, \mathrm{~d}=2.097[95 \% \mathrm{CI}=0.744-3.451])$ groups, which did not differ from each other. The three groups obtained similar results in all the cognitive tasks, excepting for the emotional go-no go, in which the participants of the ED2 group were more accurate than the control group $(\mathrm{p}=.005, \mathrm{~d}=0.766[95 \% \mathrm{CI}=0.188-1.344])$. Conversely, the ED1 group did not differ from either the ED2 or control groups (Table 1).

The EFA on DERS subscales extracted two factors that account for about $58 \%$ of the total variance. The DERS subscales load mainly on factor 1 ( $48.25 \%$ of the total variance): Nonacceptance (.787), Goals (.668), Impulse (.735), and Strategies (.814). Awareness and Clarity subscales load on factor $2(9.39 \% ; .667$ and .670 , respectively). The two factors are labeled difficulties in emotion response (DERS-R) and difficulties in emotion knowledge (DERS-K), respectively. Indeed, factor 1 (DERS-R) consisted of subscales measuring difficulties in emitting a response following an emotional state. Factor 2 (DERS-K) included two subscales measuring the difficulties in knowing/understanding an emotional state [33]. A composite score with the means of the DERS scales loading each factor was calculated.

Considering the self-reports scores, the pairwise comparisons show several differences among the groups. The ED1 group indicated more difficulties than the control group in four of the DERS subscales (all ps $<.05$ or less): in the Non-acceptance of Emotional Responses scale $(\mathrm{d}=1.341$ $[95 \% \mathrm{CI}=0.72-1.961])$, in the Lack of Emotional Awareness scale $(\mathrm{d}=1.433[95 \% \mathrm{CI}=0.809-$ 


\section{Running Head: COGNITIVE AND EMOTIONAL REGULATON IN EATING DISORDERS}

2.057]), in the Limited Access to ER Strategies scale $(\mathrm{d}=1.476[95 \% \mathrm{CI}=0.851-2.101])$, and Lack of Emotional Clarity scale $(\mathrm{d}=0.951[95 \% \mathrm{CI}=0.342-1.56])$. Also the ED2 group reported more difficulties than the control group in four DERS subscales (all ps <.05): in the Nonacceptance of Emotional Responses scale $(\mathrm{d}=2.158$ [95\% CI $=1.521-2.795])$, in the Difficulties Engaging in Goal-Directed Behaviour scale $(\mathrm{d}=0.956$ [95\% CI $=0.368-1.544])$, in the Impulse Control Difficulties scale $(\mathrm{d}=1.644[95 \% \mathrm{CI}=1.032-2.256])$, and in the Limited Access to ER Strategies scale $(\mathrm{d}=1.839[95 \% \mathrm{CI}=1.218-2.46])$. Moreover, the ED1 group reported fewer difficulties than the ED2 group in the Difficulties Engaging in Goal-Directed Behaviour scale $(\mathrm{d}=$ $1.022[95 \% \mathrm{CI}=0.188-1.857])$ and more problems in the Lack of Emotional Awareness scale $(\mathrm{d}$ $=1.027[95 \% \mathrm{CI}=0.193-1.862])$. Considering the DERS composite scores, the control group participants showed fewer problems than both the ED1 $(\mathrm{p}<.01, \mathrm{~d}=1.093[95 \% \mathrm{CI}=0.48-1.705])$ and the ED2 group $(\mathrm{p}<.001, \mathrm{~d}=2.078[95 \% \mathrm{CI}=1.446-2.711])$ in the DERS-R, whereas they showed fewer difficulties than the single ED1 group in the DERS-K factor $(p<.01, d=1.39[95 \%$ $\mathrm{CI}=0.768-2.012])$. Furthermore the two ED groups differ in the DERS-R, with the ED2 group showing more problems than the ED1 group $(\mathrm{p}<.05, \mathrm{~d}=1.075[95 \% \mathrm{CI}=0.236-1.914])$. A series of $t$ tests comparing the two composite scores along each group revealed that only in the ED2 group did the difficulties in the DERS-R prevail, rather than in the DERS-K [t (df 12) $=3.480, \mathrm{p}<.01$, $\mathrm{d}$ $=1.288]$.

Considering behavioural problems, the ED1 group indicated more difficulties than the control group in five Youth subscales (all ps <.05): in the Anxious/Depressed scale $(\mathrm{d}=1.234[95 \% \mathrm{CI}=$ $0.617-1.85])$, in the Withdrawn/Depressed scale $(\mathrm{d}=1.264[95 \% \mathrm{CI}=0.646-1.881])$, in the Social Problems scale $(\mathrm{d}=1.234[95 \% \mathrm{CI}=0.617-1.85])$, in the Thought Problems scale $(\mathrm{d}=$ $1.406[95 \% \mathrm{CI}=0.784-2.027])$. Finally, as expected, the control group showed significantly less internalizing problems than the ED1 group $(\mathrm{d}=2.035[95 \% \mathrm{CI}=1.285-2.2 .685])$. 


\section{Running Head: COGNITIVE AND EMOTIONAL REGULATON IN EATING DISORDERS}

Further analysis on the full ED sample taking into account the effects due to the lasting of the disorder (time) and to the different pharmacological treatments, if present, did not reveal any significant differences associate with the disorder persistence (see Figure 1). Considering pharmachological treatment participants that received a combination of antidepressants and antipsychotics showed a significantly lower performance score at $\operatorname{Symm} \_\operatorname{Span}[\mathrm{F}(3,20)=3.800, \mathrm{p}$ $\left.=.026, \eta_{2}=.363\right]$. and RSPAN $\left[\mathrm{F}(3,20)=3.895, \mathrm{p}=.024, \eta_{2}=.369\right]$ whereas participants treated with antidepressants seem to report less YSR_Somatic Complaints $\left[\mathrm{F}(3,19)=4.353, \mathrm{p}=.017, \eta^{2}\right.$ $=.407]$, YSR_Social Problems $\left[F(3,19)=.3 .888, p=.025, \eta_{2}=.380\right]$ and less difficulties in emotional recognition DERS-R $\left[\mathrm{F}(3,19)=4.303, \mathrm{p}=.018, \eta_{2}=.405\right]$.

\section{Discussion}

This study investigates the relationships between inhibition and WM, difficulties in ER and behavioural outcomes in adolescents and young women diagnosed with EDs compared to a TD group. In particular, it examines if inhibition and WM, as components of self-regulation, can be associated with difficulties in ER and specific non-adaptive behavioural outcomes. Results concerning the performance in EF tasks do not point to significant differences between the participants with ED and controls. The lack of significant differences between the clinical samples and the controls, was a non-expected result, which needs to be interpreted carefully, as the small samples may have undermined the results, but it is also possible that these specific tasks, focused on accuracy/ precision, may elicit perfectionism and attention to detail which are characteristic features of the spectrum [43]. This attitude may influence the performance in cognitive tasks producing a sort of paradox, as found with the emotional go-no-go task. The documented attention to detail /perfectionism in individuals with EDs e.g. [43] can also explain the higher accuracy shown by the older clinical sample group (ED2), that may be more inclined to pay attention to key body details and detect emotions in the pictures faster than other people and by so doing they manage to be more precise in this specific task. 
Regarding self-reported difficulties in ER, adolescents and young women with ED referred more ER difficulties than controls. These results confirm what the existing literature says about selfreported difficulties in ER in adolescence and young adulthood in the EDs spectrum [31]. In particular, the results show an interesting dissimilarity between younger and older participants with ED. ED1 group, shows a significant difference with respect to the control group, concerning the ER Knowledge factor [23]. This result is particularly interesting, meaning that age is important in developing a refined ability to distinguish and understand emotional responses. However, the difficulties in managing affect seem to persist as a characteristic feature of EDs. This prevalence and endurance of difficulties connected to the ER response are coherent with the struggles in overcoming the symptomatology of the spectrum [19]. It should be mentioned that the treatment administered to the clinical sample, with a specific "module" reserved to mindfulness practicing, could play a role. The literature documents a positive effect of mindfulness practices in gaining awareness and tools to improve the ability to understand emotional responses [45].

ER difficulties seem to be associated with behavioural maladaptive outcomes. ED1 reported more difficulties in scales related to internalizing issues, such as anxiety, depression, recursive thoughts and difficulties in managing behaviours in the social context [46]. Further analysis on larger samples that also take into account time, in terms of years of persistence, and treatment of the symptoms, are needed in order to better understand the link between these characteristics and whether it is possible to actually state a bi-directional relation between symptoms, and the connected effects on the body and the brain, e.g. [47], and individual characteristics.

Interestingly, the endurance of illness did not emerge to significantly impact results, whereas results showed a partial influence of pharmacological treatment. A combination of antidepressants and antipsychotics treatment is associated with a lower performance in WM tasks performance, whereas antidepressants seem to interact with results at specific self-report scales. 
A few limitations of this study warrant mentioning. First, the clinical sample size is not very large and the age variability is wide. Further studies are needed to better understand specific aspects of cognitive functioning. Despite these limitations, the study shows some strengths. Firstly, having a focus on adolescence and young adulthood, it takes into account a less investigated developmental period, and secondly, it adds knowledge about inhibition and WM in a population that has documented issues related to control processes.

\section{What is already known on this subject?}

A great part of the literature mostly focused on adults with EDs found impairments in WM and EF, showing an association between EDs and the reduced ability to control emotions and impulses. In fact, most of the studies that investigate control processes in EDs mainly focus on complex EF abilities in young adulthood. Emotion dysregulation seems to be an important predictor of binge eating and a core factor that may predict the onset of EDs .

\section{What does this study add?}

This study adds knowledge about specific aspects of Inhibition and WM in a less investigated developmental time in EDs, while also investigating the possible connection between these cognitive abilities and emotional and behavioural self-reported difficulties, taking into account as well complex variables such as time, in terms of duration of illness and pharmacological treatment when present.

\section{Ethics statement}

All procedures performed in this study involving human participants were in accordance with the ethical standards of the institutional and national research committee (Ethical Code of Italian Psychology Order and the Ethical Guidelines of the Italian Association of Psychology) and with the 1964 Helsinki declaration and its later amendments. At the time we collected the data no ethical 
committee was yet present to which we could refer to in our organization. Informed written consent was obtained from the participants or their parents if they were minor before data collection.

\section{Conflict of Interest:}

The authors declare that they have no conflict of interest

\section{Acknowlegments}

\section{Funding}

This work was supported by a doctoral grant and a research fellowship awarded to Chiara Malagoli by the University of Genoa and Accademia Ligure di Scienze e Lettere respectively. 


\section{Running Head: COGNITIVE AND EMOTIONAL REGULATON IN EATING DISORDERS}

\section{References}

[1] Fischer S \& Munsch S (2012). Self-Regulation in Eating Disorders and Obesity -Implications for Treatment. Verhaltenstherapie 22 158-164. doi:10.1159/000341540

[2] van Elburg A \& Treasure J (2013). Advances in the neurobiology of eating disorders. Curr Opin Psychiatry 26 556-561. doi:10.1097/YCO.0b013e328365a2e7

[3] van Zutphen L, Maier, S, Siep, N, Jacob, GA, Tüscher,O, Tebartz van Elst, L, Zeeck, A, Arntz, A, O’Connor, M.-F, Stamm, H, Hudek, M \& Joos, A (2018). Intimate stimuli result in frontoparietal activation changes in anorexia nervosa. Eat Weight Disord 24: 1155. Doi: $10.1007 / \mathrm{s} 40519-017-0474-\mathrm{x}$

[4] Donofry, S D , Roecklein, K A, Wildes, J E, Miller, M A, \& Erickson, K I (2016). Alterations in emotion generation and regulation neurocircuitry in depression and eating disorders: A comparative review of structural and functional neuroimaging studies. Neurosci BiobehavRev 68 911-927. doi:10.1016/j.neubiorev.2016.07.011

[5] Nyman-Carlsson, E, Birgegård, A, Engström, I, Gustafsson, S A \& Nevonen, L (2019). Predictors of outcome among young adult patients with anorexia nervosa in a randomised controlled trial. Eur Eat Disorders Rev 27(1): 76-85. Doi:10.1002/erv.2630

[6] Giedd, J N, Blumenthal, J, Jeffries, N O, Castellanos, F X, Liu, H, Zijdenbos, A, Paus, T, Evans, A C, \& Rapoport, L. (1999) Brain development during childhood and adolescence: A longitudinal MRI study. Nat Neurosci 2 861-863. doi: 10.1038/13158.

[7] Miyake, A, Friedman, N P, Emerson, M J, Witzki, A H, Howerter, A, \& Wager, T D (2000). The unity and diversity of executive functions and their contributions to complex "frontal lobe" tasks: A latent variable analysis. Cogn Psychol 41 49-100. doi:10.1006/cogp.1999.0734.

[8] Malagoli, C \& Usai, M C (2018a). The effects of gender and age on inhibition and working memory organization in 14- to 19-year-old adolescents and young adults. Cog Dev 45 10-23. doi:10.1016/j.cogdev.2017.10.005 
[9] Huizinga, M, Dolan, C V, \& Van der Molen, M W (2006) Age-related change in Executive Function. Developmental trends and latent variable analysis. Neuropsychologia $442017-$ 2036. doi:10.1016/j.neuropsychologia.2006.01.010.

[10] Finn, A S, Kraft, M A, West, M R, Leonard, J A, Bish, C E, \& Martin, R E (2014). Cognitive skills, student achievement tests, and schools. Psychol Sci 25 736-744 doi: $10.1177 / 0956797613516008$

[11] Romer, D, Betancourt, L, Giannetta, J M, Brodsky, N L, Farah, M, \& Hurt, H (2009) Executive cognitive Functions and impulsivity as correlates of risk taking and problem behavior in pre-adolescents. Neuropsychologia 47 2916-2926. doi:10.1016/j.neuropsychologia.2009.06.019

[12] Vitaro, F, Ferland, F, Jacques, C, Ladouceur, R (1998) Gambling, substance use, and impulsivity during adolescence. Psychol Addict Behav 12 185-194. doi:10.1037/0893164X.12.3.185

[13] Fikke, L T, Melinder, A, \& Landro, N I (2011) Executive Functions are impaired in adolescents engaging in non suicidal Self Injury. Psychol Med 41 601-610. doi: $10.1017 / \mathrm{S} 0033291710001030$

[14] Wu, M, Hartmann, M, Skunde, M, Herzog, W \& Friederich, HC,(2013) Inhibitory control in bulimic-type eating disorders. A systematic review and meta-analysis. PLoS ONE 8 e83412.doi:10.1371/journal.pone.0083412

[15] Darcy, AM, Fitzpatrick, KK, Manasse, SM, Datta, N, Klabunde, M, Colborn, D, Aspen, V, Stiles-Shields, C, Labuschagne, Z, Le, Grange, D and Lock, J (2015) Central coherence in adolescents with bulimia nervosa spectrum eating disorders Int J Eat Disord, 48: 487-493. doi:10.1002/eat.22340 
[16] Lopez, C, Tchanturia, K, Stahl, D, Treasure, J (2009) Weak central coherence in eating disorders, A step towards looking for an endophenotype of eating disorders. J Clin ExpNeuropsychol 31(1), 117-125. doi:10.1080/13803390802036092

[17] Roberts, M E, Tchanturia, K, Stahl, D, Southgate, L, \& Treasure, J (2007) A systematic review and meta-analysis of set-shifting ability in eating disorders Psychol Med 37 1075-1084. doi: $10.1017 / \mathrm{S} 0033291707009877$

[18] Kanakam, N, \& Treasure, J (2013) A review of cognitive neuropsychiatry in the taxonomy of eating disorders State, trait, or genetic? Cogn Neuropsychiatry 18 83-114. doi: $10.1080 / 13546805.2012 .682362$

[19] Brockmeyer, T, Skunde, M, Wu, M, Bresslein, E, Rudofsky,G, Herzog, W \& Friederich, HC (2014) Difficulties in emotion regulation across the spectrum of eating disorders Compr Psychiatry 55 565-571 doi:10.1016/j.comppsych.2013.12.001

[20] McClelland, M M, Ponitz, C C, Messersmith, E, \& Tominey, S (2010) Self-regulation: The integration of cognition and emotion. In R Lerner (Series Ed) \& W Overton (Vol Ed), Handbook of life-span development (Vol 1: Cognition, biology and methods, pp 509-553) Hoboken, NJ: Wil

[22] Demirci, 2018 Non suicidal self-injury, emotional eating and insomnia after child sexual abuse: Are those symptoms related to emotion regulation? J Forensic Leg Med 53: 17-21.doi : 10.1016/j.jflm.2017.10.012

[22] Rosenstein, L K, Ellison, WD, Walsh, E, Chelminski, I, Dalrymple, K, \& Zimmerman, M (2018) The role of emotion regulation difficulties in the connection between childhood emotional abuse and borderline personality features Personal Disord 9(6): 590-594. doi:10.1037/per0000294 
[23] Brockmeyer, T, Pellegrino, J, Maier C, Münch, HM, Harmer, CJ Walther, S, Herzog, W \& Friederich, HC (2019) Blunted emotion-modulated startle reflex in anorexia nervosa Int J Eat Disord 52(3):270-277. doi: 10.1002/eat.23022

[24] Foye, U, Hazlett, DE \& Irving, P (2019) Exploring the role of emotional intelligence on disorder eating psychopathology. Eat Weight Disord 24: 299 doi: 10.1007/s40519-018-06294

[25] Laghi, F, Bianchi, D, Pompili, S, Lonigro, A, \& Baiocco, R., (2018). Metacognition, emotional functioning and binge eating in adolescence: the moderation role of need to control thoughts. Eat Weight Disord 23(6): 861-869. doi:10.1007/s40519-018-0603-1

[26] Oldershaw, A \& Lavender, T \& Sallis, H\& Stahl, D \& Schmidt, U (2015) Emotion generation and regulation in anorexia nervosa: A systematic review and meta-analysis of self-report data. Clin Psychol Rev. 39. doi: 10.1016/j.cpr.2015.04.005.

[27] Segal, A \& Golan, M (2016) Differences in Emotion Regulation along the Eating Disorder Spectrum: Cross Sectional Study in Adolescents out Patient Care. J Psychol Clin Psychiatry 6(1): 00314. doi: 10.15406/jpcpy.2016.06.00314

[28] Stice, E (2002) Risk and Maintenance Factors for Eating Pathology: A Meta-Analytic Review Psychol Bull 128, 825-848 doi: 10.1037//0033-2909.128.5.825

[29] Whiteside, U, Chen, E, Neighbors, C, Hunter, D, Lo, T, \& Larimer, M. (2007). Difficulties regulating emotion: do binge eaters have fewer strategies to modulate and tolerate negative affect? Eat Behav 8 162-169. doi:10.1016/j.eatbeh.2006.04.001

[30] Aldao, A, Nolen-Hoeksema, S, \& Schweizer, S (2010). Emotion-regulation strategies across psychopathology: a meta-analytic review. Clin Psychol Rev 30 217-237. doi: 10.1016/j.cpr.2009.11.004. 
[31] Goossens, L, Van Malderen, E, Van Durme, K, \& Braet, C (2016). Loss of control eating in adolescents: Associations with adaptive and maladaptive emotion regulation strategies. Eat Behav 22 156-163. doi:10.1016/j.eatbeh.2016.06.015

[32] Adambegan, M, Wagner, G, Nader, I W, Fernandez-Aranda, F, Treasure, J, \& Karwautz, A (2012) Internalizing and externalizing behaviour problems in childhood contribute to the development of anorexia and bulimia nervosa — a study comparing sister pairs Eur Eat Disord Rev 20 116-120. doi:10. 1002/erv.1152

[33] Malagoli, C\& Usai, M C (2018b) WM in Adolescence: What is the Relationship With Emotional Regulation and Behavioral Outcomes? Front Psychol 9844. doi:10.3389/fpsyg.2018.00844

[34] Logan, G D (1994) On the ability to inhibit thought and action: A user's guide to the stop signal paradigm. In D Dagenbach, \& T H Carr (Eds) Inhibitory processes in attention, memory, and language (pp. 189-239). San Diego, CA: Academic Press.

[35] Verbruggen, F, \& Logan, G D (2008). Response inhibition in the stop-signal paradigm. Trends Cogn Sc 12 418. doi:10.1016/j.tics.2008.07. 005.

[36] Donders, F C (1969) On the speed of mental processes. Acta Psycho 30 12-431

[37] Hare, T A, Tottenham, N, Davidson, M C, Glove, G H, \& Casey, B J (2005) Contributions of amygdala and striatal activity in emotion regulation. Biol Psychiatry 57 624-32. doi:10.1016/j.biopsych.2004.12.038

[38] Kane, M J, Hambrick, D Z, Tuholski, S W, Wilhelm, O, Payne, T W, \& Engle, R W (2004) The generality of working memory capacity: A latent-variable approach to verbal and visuospatial memory span and reasoning. J Exp Psychol Gen 133 189-217. doi:10.1037/0096-3445.133.2.189.

[39] Daneman, M, \& Carpenter, P A (1980) Individual differences in working memory and reading. J Verbal Learning Verbal Behav 19 450-466. doi:10.1016/S0022-5371(80)90312-6. 
[40] Giromini, L, Velotti P, de Campora, G, Bonalume, L, Zavattini, G C (2012) Cultural adaptation of the difficulties in emotion regulation scale: Reliability and validity of an Italian version. J Clin Psychol 68 989-1007. doi:10.1002/jclp.21876

[41] Achenbach,T (2001) YSL ages 11-18, ASEBA, University of Vermont (USA). Italian version by A Frigerio (Ed.).

[42]. Riniolo, TC (1999) Using a Large Control Group for Statistical Comparison: Evaluation of a Between-Groups Median Test, J Exp Educ 68:1 75-88, doi: 10.1080/00220979909598495

[43] Del Pozo, A, Harbeck, S, Zahn, S, Kliem, S, \& Kröger , C (2018) Cognitive distortions in anorexia nervosa and borderline personality disorder. Psychiatry Res 260164-172. doi:10.1016/j.psychres.2017.11.043

[44] Johnston, J, Shu, CY, Hoiles, K J, Patrick J F Clarke, P J F, Watson, H J, Dunlop, P D \& Egan, S J (2018) Perfectionism is associated with higher eating disorder symptoms and lower remission in children and adolescents diagnosed with eating disorders Eat Behav 30 55-60. doi:10.1016/j.eatbeh.2018.05.008

[45] Katterman, S N, Kleinman B M, Hood, M M, Nackers L M, \& Corsica J A (2014) Mindfulness meditation as an intervention for binge eating, emotional eating, and weight loss: a systematic review.Eat Behav 15 197-204. doi:10.1016/j.eatbeh.2014.01.005.

[46] Doba, K, Berna, G, Constant, E, \& Nandrino, J L (2018) Self-differentiation and eating disorders in early and middle adolescence: A cross-sectional path analysis.Eat Behav 29 7582. doi:10.1016/j.eatbeh.2018.03.003

[47] Kaye, W H, Frank, G K, Bailer, U F, \& Henry, S E (2005) Neurobiology of anorexia nervosa: clinical implications of alterations of the function of serotonin and other neuronal systems.Int J Eat Disord 37 S15-S19. doi:10.1002/eat.20109 
[48] Di Bernardo, M, Barciulli, E, Ricca, V, Mannucci, E, Moretti, S, Cabras, PL \& Rotella, C M(1998) Binge eating scale in obese patients: Validation of the Italian version. Minerva Psichiatrica 39:125-130. doi:10.1007/s11695-011-0537-4

[49] Cuzzolaro, M, Vetrone, G, Marano, GF \& Battacchi, MW (1999) BUT: una nuova scala per la valutazione del disagio relativo all'immagine del corpo. Psichiatria dell'Infanzia e dell'adolescenza 66:417-28

[50] Cuzzolaro, Massimo \& Vetrone, G \& Marano, G \& Garfinkel, Paul (2006) The Body Uneasiness Test (BUT): Development and validation of a new body image assessment scale.Eat Weight Disord 11 1-13.doi: 10.1007/BF03327738.

[51] Calugi, S, Sartirana, M, Milanese, C, El Ghoch, M, Riolfi, F, \& Dalle Grave, R (2018) The clinical impairment assessment questionnaire: validation in Italian patients with eating disorders.Eat Weight Disord 23: 685 doi:10.1007/s40519-018-0477-

[52 ]Garner, D M, Olmsted, M P, \& Polivy, J (1983) Development and validation of a multidimensional EDI for anorexia nervosa and bulimia Int J Eat Disord 2, 15-34.

[53] Giannini M, Pannocchia L, Dalle Grave R, Muratori F, Viglione V EDI-3 Eating Disorder Inventory-3: Manuale. Firenze: O.S. Organizzazioni Speciali, 2008.

[54] Bressi, C, Taylor, G, Parker, J, Bressi, S, Brambilla, V, Aguglia, E, Invernizzi, G (1996) Cross validation of the factor structure of the 20-item Toronto Alexithymia Scale: An Italian multicenter study. J Psychosom Res 41(6), 551-559. https://doi.org/10.1016/S00223999(96)00228-0

[55] Sarno, I, Preti, E, Prunas, A, \& Madeddu, F (2011) SCL-90-R Symptom Checklist-90-R Adattamento italiano Firenze : Giunti, Organizzazioni Speciali. 